\title{
Osteoporosis-oculocutaneous hypopigmentation syndrome
}

INSERM

\section{Source}

INSERM. (1999). Orphanet: an online rare disease and orphan drug data base.

Osteoporosis-oculocutaneous hypopigmentation syndrome. ORPHA:2786

Osteoporosis-oculocutaneous hypopigmentation syndrome is characterised by osteoporosis and congenital oculocutaneous hypopigmentation. Three cases have been described in the literature. The mode of inheritance appears to be autosomal recessive. 